\section{Krebs und Psyche - Psychoonkologie, Hattingen, März 2001}

Die Modellabteilung für Naturheilkunde der Klinik Blankenstein veranstaltete gemeinsam mit dem Psychosozialen Zentrum Sprockhövel im März 2001 in Hattingen-Blankenstein eine Tagung für Ärzte, Psychologen, Psychotherapeuten, Betroffene und interessierte Laien zum Thema «Krebs und Psyche».

Einführend stellte A.-M. Beer, Hattingen, Ziele und Inhalte der Psychoonkologie vor. Psychoonkologie versteht sich als die Lehre von den psychosozialen Begleitumständen einer Krebserkrankung. Die Praxisfelder der Psychoonkologie sind die Prävention, die Akutbehandlung und die Nachsorge.

Seit gut 25 Jahren gibt es in Deutschland psychoonkologische Aktivitäten, seit 1988 ist die Psychoonkologie als offizielle Arbeitsgemeinschaft (PSO) in der Deutschen Krebsgesellschaft vertreten. Bis dahin war es jedoch ein weiter Weg: Zum körperlichen kam seit jeher das psychische Leid der Patienten. Beer verdeutlichte dies am Beispiel des Mammakarzinoms. Das Wissen um die stets wechselnden therapeutischen Vorgehensweisen bei der Behandlung von Brustkrebs und den damit für die Betroffenen verbunden psychischen Belastungen lässt die Entwicklung hin zur Psychoonkologie besser verständlich werden.

Das Mammakarzinom wurde 1894 von Rotter und Halsted als lokale Erkrankung definiert. Dies basierte auf den Vorstellungen von Rudolf Virchow, der die Erkrankung des Körpers noch als Erkrankung der einzelnen Zelle sah. Dies führte zu der Konsequenz, dass man das Mammakarzinom folgerichtig möglichst radikal operierte: Man führte eine sogenannte Ablatio mammae durch, entfernte den grossen und kleinen Brustmuskel und entnahm die interkostalen Lymphknoten. Eine Frau wurde dadurch aus verschiedenen Gründen gesellschaftsunfähig. Sie musste z. B. die Haare lang tragen, um die Narben zu verhüllen, was um die Jahrhundertwende als Armutszeichen galt und bekam so häufig Probleme mit ihrem sozialen Umfeld. Die Namen Rotter und Halsted werden heute noch als ein Synonym für die sogenannte radikale Mastektomie benutzt.

Die Amerikaner Patey und Dyson (1965) gelten als Begründer der sogenannten eingeschränkten radikalen Mastektomie. Sie operierten unter Erhalt des grossen Brustmuskels. Ihnen kommt insofern besonderer Verdienst zu, als dass sie erstmals das grosse Leid der Frau, besonders nach Operationen, erkannt haben. Sie definierten das Mammakarzinom als eine systemische, also den ganzen Körper betreffende Erkrankung, wonach nicht nur eine lokale, sondern vor allem eine systemische Therapie erforderlich wurde.

In der Folge stufte Haybittle (1985) das Mammakarzinom nicht nur als systemische, sondern auch als chronische Erkrankung ein. Damit versuchte er der Erfahrung gerecht zu werden, dass bis zu 10 Jahre nach der Ersterkrankung eine Progression und eine Metastasierung erfolgen kann.

Beer verdeutlichte, dass auch heute die wirksame Therapie des Mammakarzinoms noch nicht bekannt sei. Eine «objektive verbindliche Therapie» sei bislang nicht gefunden worden. Eine echte Heilung des Mammakarzinoms gebe es daher in absehbarer Zeit nicht. Gerade darum sei auch der Patientenwille für den Therapieerfolg ausschlaggebend. Beer schloss, dass eine Optimierung der Lebensqualität nur unter Zuhilfenahme klassischer Naturheilverfahren objektiv möglich und machbar sei. Aus den oben genannten Ausführungen wird deutlich, dass Betroffene neben der bestmöglichen medizinischen Behandlung auch eine angemessene seelische Betreuung benötigen, um der Krebserkrankung optimal zu begegnen. Hierzu bietet sich eine psychoonkologisch orientierte Ordnungstherapie an. Sie zählt zu den klassischen Naturheilverfahren.

Der Begriff Ordnungstherapie wurde vom Schweizer Naturarzt Bircher-Benner geprägt und umfasst alle therapeutischen Bemühungen, der natürlichen Ordnung der Lebensvorgänge, in Prävention, Therapie und Rehabilitation Rechnung zu tragen. In Amerika wird an der Harvard-Universität die sogenannte Mind-Body-Medizin gelehrt. Dies meint ebenfalls das «Sich-in-Ordnung-Bringen».

In seinem Referat stellte S. Fey, Hattingen, Facharzt für Allgemeinmedizin, die klassischen Naturheilverfahren, wie sie bei onkologischen Patienten in der Modellabteilung für Naturheilkunde stationär durchgeführt werden, dar. Auch er merkte an, dass die Ordnungstherapie das wesentliche Fundament aller naturheilkundlichen Therapien bei malignen Erkrankungen sei. Ziel der naturheilkundlichen Massnahmen sind die Stärkung der Selbstheilungskräfte und die Erhaltung bzw. Erhöhung der Lebensqualität.

Die Ordnungstherapie ist eine der fünf Säulen der klassischen Naturheilverfahren, zusammen mit Kneipp'scher Therapie, Phytotherapie (Pflanzenheilkunde), Bewegungstherapie und Ernährungstherapie.

Die Auswirkungen bösartiger Tumoren auf die psychosoziale Situation des Betroffenen rückt immer mehr in den Vordergrund und beinhaltet Ängste, depressive Verstimmungen, den Umgang mit Schmerzen, Übelkeit und Essstörungen sowie die

\section{KARGER}

Fax +497614520714

E-mail Information@Karger.de

www.karger.com
(C) 2001 S. Karger GmbH, Freiburg

Accessible online at:

www.karger.com/journals/fkm 
Auseinandersetzung mit Krankheit und Tod. Dabei spielt bei allen therapeutischen Massnahmen die Lebensqualität des Patienten eine bedeutende Rolle. Naturheilkundliche Therapien sind jedoch keine Alternative zur schulmedizinischen Behandlung, sondern eine sinnvolle Ergänzung.

Das Hauptreferat des Abends hielt F. Neuendorff, Psychotherapeut und Theologe, der in der Modellabteilung für Naturheilkunde der Klinik Blankenstein, Hattingen, psychoonkologisch schwerpunktmässig tätig ist. Sein Vortrag lässt sich in drei Themenbereiche gliedern:

1. Krebs und Psyche: Krebs ist mehr als nur ein Tumor. Krankheit und die Behandlungsfolgen betreffen Körper und Seele, die erkrankten Zellen ebenso wie unsere innersten Gefühle oder das Zusammenleben in der Familie. Psychoonkologie versucht, Patienten zu einer eigenverantwortlichen Krankheitsbewältigung zu verhelfen und so die Lebensqualität $\mathrm{zu}$ verbessern. Dabei bezieht sie sich nicht nur auf die Erkrankten, sondern auch Familie, soziales Umfeld und das medizinische Team.

2. Psyche und Krebs: Der Einfluss der Psyche auf Entstehung und Behandlung einer Krebserkrankung ist umstritten. Wissenschaftliche Studien scheinen aber zu zeigen, dass eine Untersuchung der Wechselwirkungen verschiedener Risikofaktoren eine wichtige Rolle spielt. Hierbei existieren Faktoren, die wie Katalysatoren das System in Richtung Krankheit oder Gesundheit dirigieren. Dies sind vor allem die genetische Disposition und psychischer Stress, der durch eine gehemmte Selbstregulation entstehen kann. Selbstregulation ist dabei die Fähigkeit, durch kompetente Eigenaktivität ein hohes Mass an Wohlbefinden, Lust und Sicherheit zu erreichen, wobei die jeweiligen psychosozialen Umweltbedingungen kompetent mit einbezogen werden sollen. Ist die Fähigkeit zur Selbstregulation stark ausgeprägt, wird die Lebenstendenz von Menschen sowohl seelisch als auch körperlich positiv angeregt. Statistisch gesehen leben diese Personen länger, werden weniger krank oder haben zumindest deutlich bessere Chancen, etwa eine Krebserkrankung erfolgreich zu bewältigen. Ist die Selbstregulation dagegen gehemmt, fühlen sich Menschen hilflos und hoffnungslos im Blick auf ihre zentralen Bedürfnisse und Lebensträume, so kann es zu einer «Todestendenz» des ganzen Systems kommen. Das Risiko, an Krebs oder Herzinfarkt zu erkranken, steigt deutlich an, gleichzeitig werden die Chancen deutlich schlechter, eine solche Krankheit zu überwinden.

3. Folgerungen für die Ordnungstherapie: Die Ordnungstherapie versucht, gemeinsam mit anderen Bereichen der Naturheilkunde, den Patienten zu unterstützen und die körperlichen und seelischen Folgen einer Krebserkrankung eigenverantwortlich und angemessen zu bewältigen. Zentrales Anliegen ist es darüber hinaus, den Patienten darin zu unterstützen, seine zentralen Lustquellen und Lebensbedürfnisse neu oder wiederzuentdecken und in kompetenter Eigeninitiative in seinem Leben neuen Raum zu geben. Ordnungstherapie in diesem Sinne beschränkt sich demnach nicht darauf, Patienten in ihrer Erkrankung und seelischen Not lediglich zu begleiten. Ordnungstherapie hilft, eigenverantwortlich Ordnung in das Leben zu bringen, um damit mehr Lebensqualität und einen befriedigenden Gesundheitszustand zu erreichen.

In der anschliessenden Diskussion zeigte sich, dass Psychoonkologie heute noch kein überall fest etabliertes Lehrfach darstellt. Psychoonkologische Aspekte sind heute zwar ein fester Bestandteil der Krebsbehandlung, wobei jedoch die institutionelle Umsetzung (= feste Stellen hierfür) regional sehr unterschiedlich ist.

Die Tagung hat allseits reges Interesse gefunden. Die notwendige Arbeitsatmosphäre wurde durch die Klinik Blankenstein geschaffen. Unter der Schirmherrschaft des Landtagspräsidenten von Nordrhein-Westfalen, Herrn U. Schmidt, wurde diese Tagung für alle Zuhörer zu einem interessanten Ereignis. Diese Veranstaltung zeigt einmal mehr, dass künftig Fortbildung für Fachleute und Informationsveranstaltungen für Laien zum Thema Psychoonkologie verstärkt erforderlich sind. 